\title{
Stress echocardiogaphy: A useful test for assessing cardiac risk in diabetes
}

\author{
Rajan Sharma' \\ Denis Pellerin ${ }^{2}$ \\ 'Ealing Hospital, London, UK; \\ ${ }^{2}$ The Heart Hospital, London, UK
}

\begin{abstract}
Cardiovascular disease is the leading cause of death in patients with diabetes therefore prompt diagnosis and treatment of high risk groups is essential. Dobutamine stress echocardiography is a safe, cheap and reliable method for coronary artery disease diagnosis and provides important long term prognostic information. The diagnostic accuracy and prognostic power of this technique has been validated by a number of studies on patients with and without diabetes. This article discusses the application, interpretation, and accuracy of stress echocardiography both in the general population and in patients with diabetes. The role of advanced imaging modalites such as myocardial contrast agents and tissue Doppler imaging is also explored.
\end{abstract}

Keywords: diabetes, dobutamine stress echocardiography, ischemia, cardiovascular risk

\section{Introduction}

Patients with diabetes mellitus have a significantly increased risk of ischemic cardiovascular mortality and morbidity. ${ }^{1-3}$ Diagnosis can be difficult as the symptoms are often atypical and significant obstructive coronary disease clinically silent. This is thought to be in part due to abnormal perception of angina caused by autonomic neuropathy. Dobutamine stress echocardiography (DSE) is a widely used tool for both ischemic heart disease diagnosis and risk stratification in different clinical settings. This article highlights the principles and practice of this technique and its efficacy in diabetic patients.

\section{Pathophysiology: The ischemic cascade}

Myocardial ischemia is caused by a transient imbalance between oxygen supply and demand, the signs and symptoms of which can be used as a diagnostic tool. A typical cascade of events in a well defined time sequence will occur after the onset of ischemia. ${ }^{4}$ The forerunner is abnormal myocardial perfusion and endothelial dysfunction, followed by regional wall motion dyssynergy, and only at a later stage by electrocardiography (ECG) changes and pain. DSE aims to detect regional wall motion abnormalities under ischemic conditions and thus allows diagnosis at an earlier stage of the cascade than would be achieved by symptoms or ECG changes (Figure 1).

\section{Methodology The stress agent}

Exercise, either via bicycle or treadmill, is the most attractive stress agent as it also provides important physiological data. However, this mode of stress is limited in patients unable to exercise and requires very rapid acquisition of the echo images. Consequently, pharmacological stress is often preferred. Coronary vasodilators such as adenosine and dipridamole cause a relative hyperemia with coronary steal to induce ischemia. However, they cause relatively less effects on wall motion during stress and are less accurate 


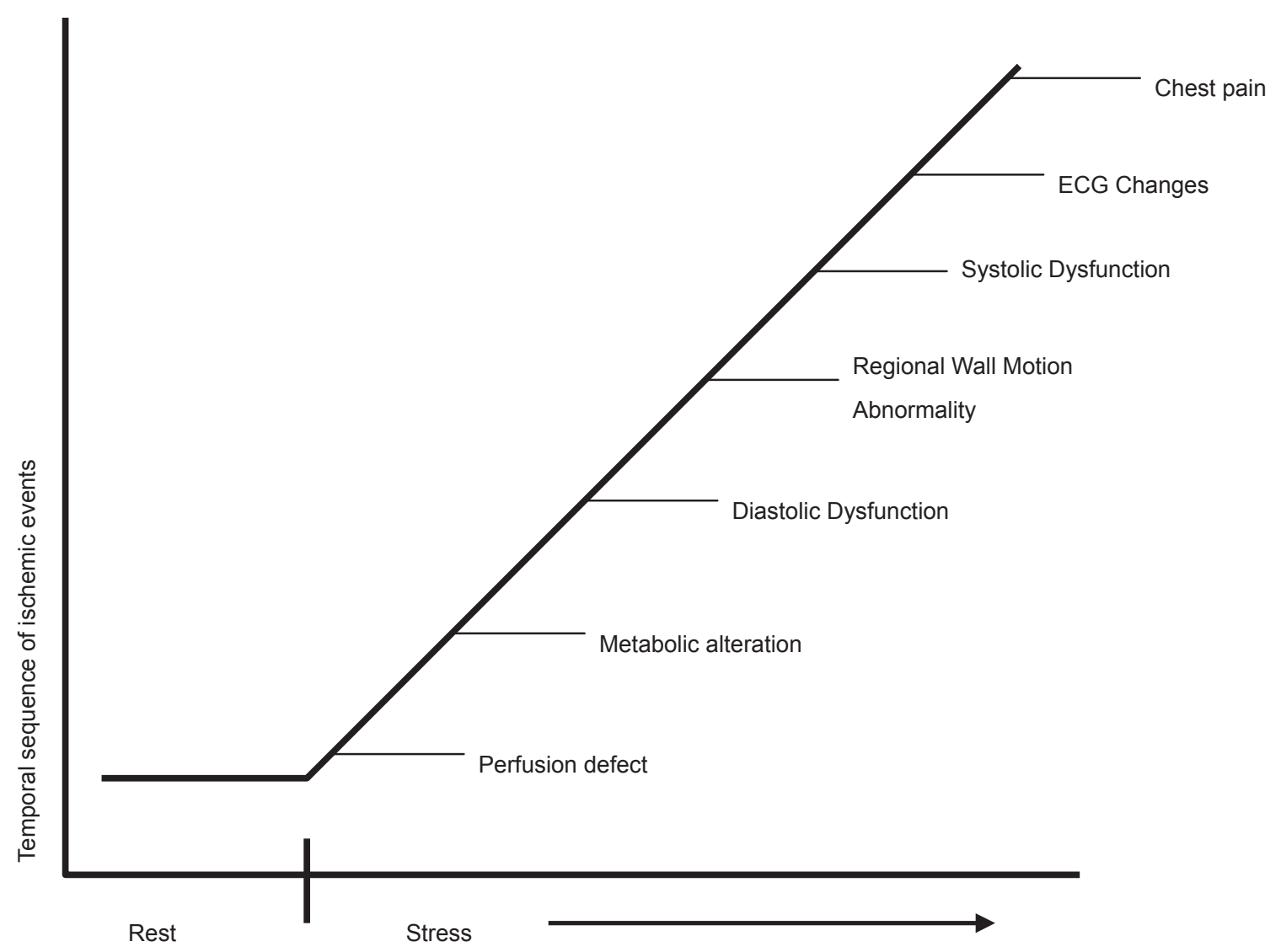

Figure I The ischemic cascade.

than inotropic agents such as dobutamine. Thus dobutamine remains the pharmacological stress agent of choice in most institutions. Pacing induces ischemia by increasing myocardial oxygen consumption (due to increased heart rate) and reducing myocardial subendocardial perfusion. However, the technique is invasive and involves atrial stimulation via the transvenous or transoesophageal route so is rarely used.

\section{Image acquisition}

Dobutamine is the most often used stress agent as discussed above. Ischemia is provoked mainly via the enhanced chronotropic and inotropic response to stimulation. Transthoracic echo images of the heart are acquired in standard parasternal long- and short-axis and apical 2-, 3-, 4-chamber views at baseline and during stepwise infusion of dobutamine. This is given according to a protocol based on 3 minute stages of $5,10,20,30,40 \mu \mathrm{g} / \mathrm{kg} / \mathrm{min}$. Atropine can be administered up to a total of $1.0 \mathrm{mg}$ intravenously if the target heart rate is not achieved with dobutamine alone. Blood pressure and 12-lead ECG are recorded at baseline and at each infusion stage. Baseline, low-dose (heart $10-15$ beats above baseline), peak and recovery (10 minutes after drug infusion terminated) stage images are acquired as digital loops in a quad screen format and stored for off-line analysis. The test is stopped if: target heart rate is achieved ([220 - age] $\times 0.85)$, ST depression $>2 \mathrm{~mm}$, significant tacharrhythmia, symptomatic severe hypotension, blood pressure $>240 \mathrm{mmHg}$ systolic or $140 \mathrm{mmHg}$ diastolic. Serious side effects are rare (1:1000 patients). ${ }^{5}$ Common minor side effects include headache, nausea, shivering, urgency, hypotension. Because of the short half life of dobutamine ( 2 minutes,) there is rapid resolution of its effects once the infusion has been stopped.

\section{Interpretation of stress echocardiography}

Interpretation is usually by visual assessment based on analysis of thickening and inward systolic motion before, during and after stress. The heart is typically divided into a 17 segment model $^{6}$ (Figure 2) and a score assigned to each segment at baseline and during stress based on the degree of thickening as shown in Table 1. ${ }^{7}$ The presence of a new or worsening regional wall motion abnormality in 1 or more segments identifies an ischemic response. The site of this response gives a clue as to the artery affected (Figure 2). The extent of ischemia is based on the number of affected segments, the occurrence of ischemia 


\section{Balanced Circulation}
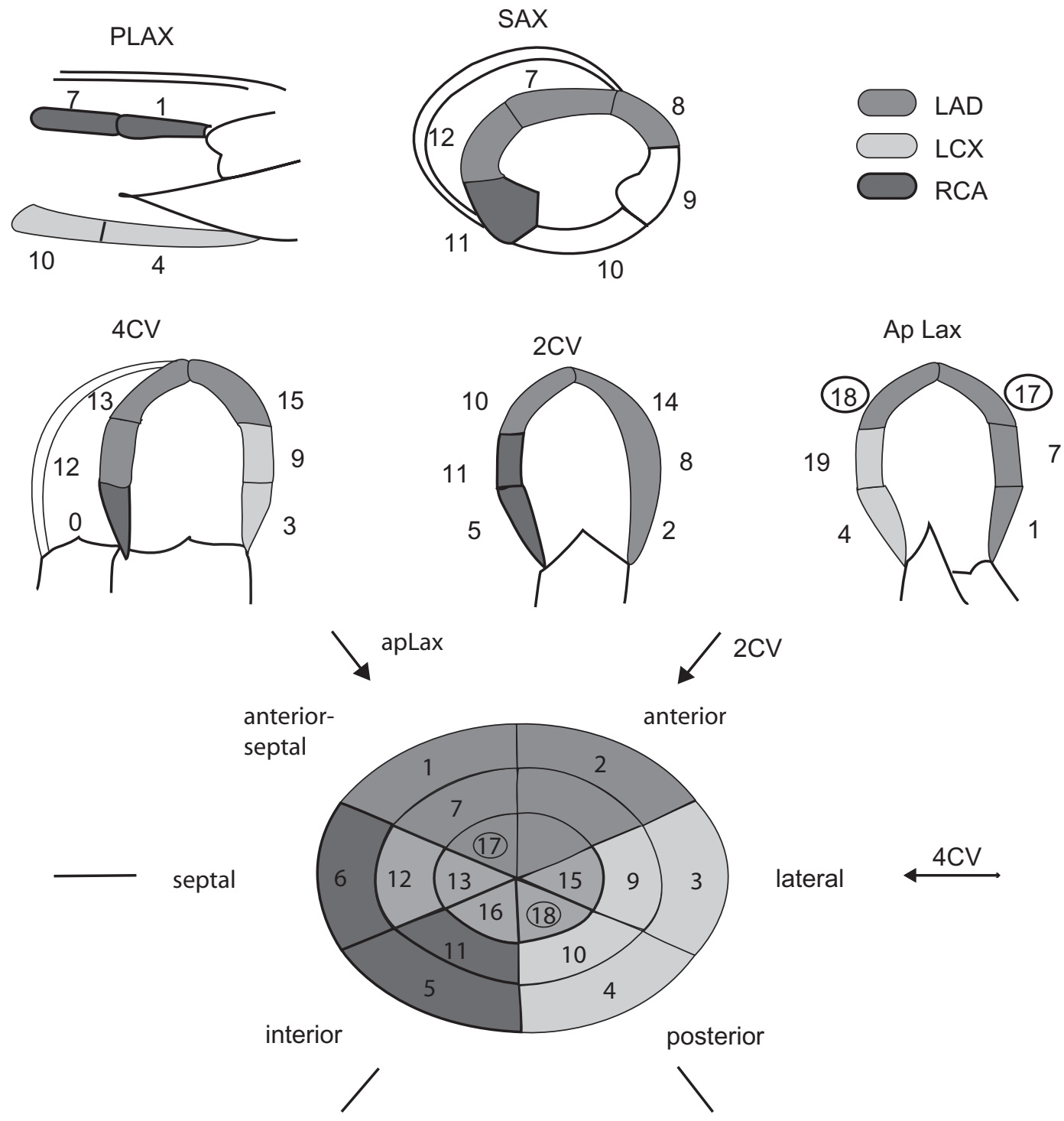

Figure 2 17-segment cardiac model.

at an early stage of the test, ${ }^{8}$ the wall motion score index at rest and peak stress, ${ }^{9}$ and a slow recovery time. ${ }^{10}$ Global left ventricular (LV) ejection fraction and end systolic volumes may also be calculated at baseline and at peak stress. A fall in either of these parameters is an indicator of significant ischemia.

This qualitative approach requires significant training ${ }^{11}$ and there are problems with reproducibility in inexperienced hands. ${ }^{12}$ Five percent to $10 \%$ of patients will have poor acoustic windows such that insufficient cardiac segments can be analyzed.

\section{Accuracy of stress echocardiography}

The accuracy of this technique is consistently reported to be good with sensitivity and specificity averaging $82 \%$ and
$81 \%$, respectively. ${ }^{13}$ This is comparable to perfusion imaging and superior to exercise ECG. As with other forms of stress testing, the accuracy is lower in those with single vessel disease. ${ }^{14}$ Accuracy is also reduced in patients taking beta blockers although this can be overcome by administration of atropine to achieve the target heart rate. Advances in image acquisition, analysis, and interpretation have enhanced the accuracy and prognostic value of the test. Nevertheless, the technique is subjective and interinstitutional observer agreement remains suboptimal despite these advances. ${ }^{15}$ In assessing the relative merits of which test to select for coronary artery disease (CAD) diagnosis in any given institution, the overriding consideration must always be local expertise. 
Table I The segmental scoring system

\section{Score:}

I = Normal/Hyperkinetic: normal/increased systolic wall motion and thickening

2 = Hypokinetic: decreased systolic wall motion and thickening

$3=$ Akinetic: absent systolic wall motion and thickening

4 = Dyskinetic: outward systolic wall motion and thickening

Notes: Wall Motion Score Index, Total score of analyzed segments/number segments analyzed.

\section{Prognostic evaluation}

A normal stress echo carries a very low risk ( $<1 \%$ per year) of major cardiac events in the subsequent 5 years. ${ }^{16} \mathrm{~A}$ positive stress test carries a $10 \%-30 \%$ risk of further cardiac events, with risk being greatest in those with the highest ischemic burden. ${ }^{17}$ The prognostic value of DSE is independent and additive to resting echo and exercise ECG. ${ }^{18}$

\section{Clinical application}

Table 2 lists the major uses of DSE in clinical cardiology. In all of the above settings the test gives diagnostic and prognostic information. A detailed account of its role in viability and valvar heart disease is outside the scope of this review article. It is being increasingly used to risk stratify patients with no cardiac symptoms awaiting high risk non cardiac surgery. This is especially true of patients awaiting major vascular surgery ${ }^{19}$ and in renal transplant candidates, ${ }^{20}$ where there is a high probability of underlying CAD.

\section{New technologies in stress echocardiography Myocardial contrast agents}

Left ventricular opacification with contrast agents injected peripherally greatly enhances endocardial border definition. ${ }^{21}$ This now allows an accurate diagnosis in patients previously considered not suitable for DSE due to poor image quality. ${ }^{22}$ Assessment of myocardial perfusion using contrast at rest and under stress is also now possible. ${ }^{23}$ This technique is promising because a perfusion abnormality would be expected to occur before a regional wall motion abnormality in the ischemic cascade However, at present its use is limited to a few centers only and further standardization of the methodology is required before the technique is established.

\section{Coronary flow reserve}

Doppler echocardiographic-derived coronary flow reserve (CFR) has been studied in diabetic and nondiabetic patients. The technique is especially reproducible for the left anterior descending artery. Using a CFR cut off value of $<$ or $=2$, CFR provides independent prognostic information in diabetic patients with $\mathrm{CAD}$ and a negative dipyridamole stress echocardiogram. ${ }^{24}$

\section{Tissue Doppler and strain rate imaging}

Tissue Doppler imaging allows assessment of myocardial motion in preference to blood flow. Global and regional systolic and diastolic velocities can be displayed at rest and under stress. The study can thus be quantified. During ischemia, systolic and diastolic velocities are reduced in amplitude with a delayed timing of peak contraction after aortic valve closure: post systolic thickening (Figure 3). ${ }^{25}$ However, this technique remains angle-dependent and velocities in a given segment of the heart are influenced by tethering to adjacent segments and rotational motion of the myocardium under stress. This problem can be overcome by assessing deformation of the myocardium between 2 segments: strain rate imaging. ${ }^{26}$ While these techniques may improve the diagnostic accuracy of stress echo and reduce interobserver variability, their use requires considerable expertise and machines with high frame rates.

\section{Dobutamine stress echocardiography in diabetes}

Approximately $50 \%$ of mortality in diabetic patients is related to $\mathrm{CAD} .{ }^{27}$ Risk of death from cardiovascular disease in this population is equivalent to nondiabetics after myocardial infarction. ${ }^{28}$ Assessing cardiovascular risk in diabetic patients is therefore crucial. Sex, lipid levels, ${ }^{29}$ resting ST segment changes are less predictive in diabetes and silent ischemia is more common. ${ }^{30}$ Associated peripheral vascular disease and poor exercise capacity make standard exercise ECG testing in this group less effective. DSE is therefore an attractive method of assessing cardiovascular risk in diabetics.

\section{Prognostic evaluation of dobutamine stress echo in diabetes}

Several studies have shown stress echo is a feasible and safe in diabetic patients. ${ }^{31-34}$ The accuracy is the same as for nondiabetics. The presence of inducible regional wall motion abnormality under stress is an independent predictor of death, incremental to other factors, especially resting LV function. ${ }^{32,34}$ A negative stress echo result is associated with a higher mortality ( $4 \%$ per year) than a 
Table 2 Indications for dobutamine stress echo

I. Patients unable to undergo an exercise test or with an inconclusive result due to resting electrocardiogram abnormalities or submaximal heart rate achieved.

2. Functional significance of an angiographically defined stenosis.

3. Risk assessment for major non cardiac surgery.

4. Long term prognosis after acute myocardial infarction.

5. Viability assessment for contractile reserve in those with poor left ventricular function referred for revascularisation.

6. Loss of contractile reserve in asymptomatic patients with severe valvular regurgitation and normal left ventricular dimensions.

7. Identification of patients who may benefit from surgery with low flow, low gradient aortic stenosis.

similar result in nondiabetics ( $1 \%$ per year). The diabetics who die despite a normal stress echo tend to be older, have poorer exercise capacity, and have poorer LV function at rest. A positive stress echo result in a diabetic is associated with a much higher annual mortality ( $10 \%$ per year) than a similar result in a nondiabetic (5\% per year). Those with impaired LV systolic function at rest and a high ischemic burden are at greatest risk..$^{32,34}$

A positive stress ECG is a marker of adverse outcome in asymptomatic diabetics. It is also a useful tool for identifying high cardiac risk renal transplant candidates. ${ }^{35}$ In patients undergoing major vascular surgery, unrecognized myocardial infarction and silent myocardial ischemia, detected by stress echocardiography, are highly prevalent (23\% and $28 \%$ respectively) and are associated with increased long term cardiovascular events. ${ }^{36}$

The timing of serial stress studies in diabetic patients with an initial normal stress test is also uncertain. Elhendy and colleagues ${ }^{33}$ have suggested that the cardiac event rate is low within 2 years after a stress echo, but after this events may occur.

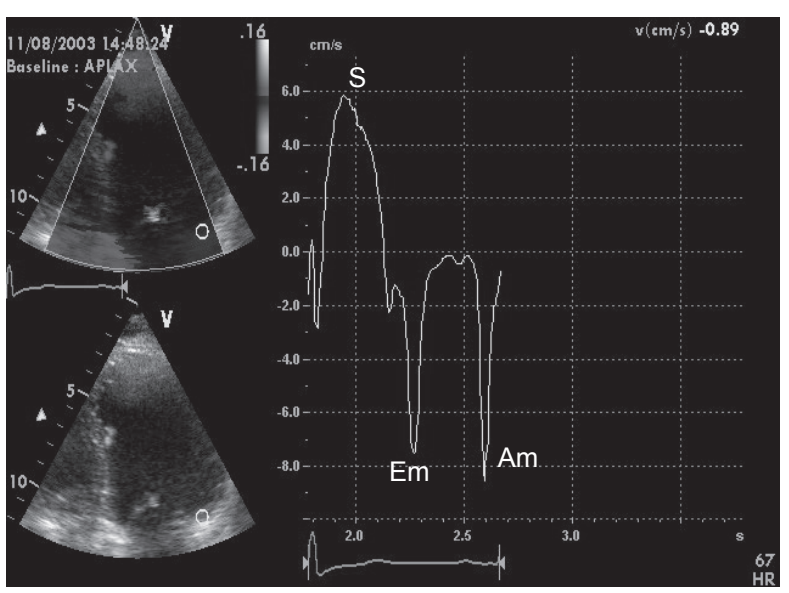

Baseline

\section{Other techniques for detection of coronary disease in diabetics}

Nonspecific ST and T wave changes on the 12 lead ECG are strong predictors of inducible ischemia in asymptomatic diabetic patients. A high coronary calcium score (>400 Agaston units) from cardiac computed tomography (CT) is associated with a high incidence of inducible ischemia in diabetic patients. ${ }^{37}$ Myocardial perfusion imaging also has excellent accuracy for $\mathrm{CAD}$ diagnosis and event prediction in diabetics. ${ }^{38} \mathrm{~A}$ normal study is associated with a low event rate within the next 2 years. Multislice CT coronary angiography, stress magnetic resonance imaging (MRI) and MRI perfusion are emerging techniques for CAD detection, but have not been extensively investigated in the diabetic population.

\section{Screening for cardiovascular disease in diabetes}

Advocating which diabetic patients should be investigated for cardiovascular disease remains difficult. Only $5 \%-10 \%$ of asymptomatic diabetics will have obstructive

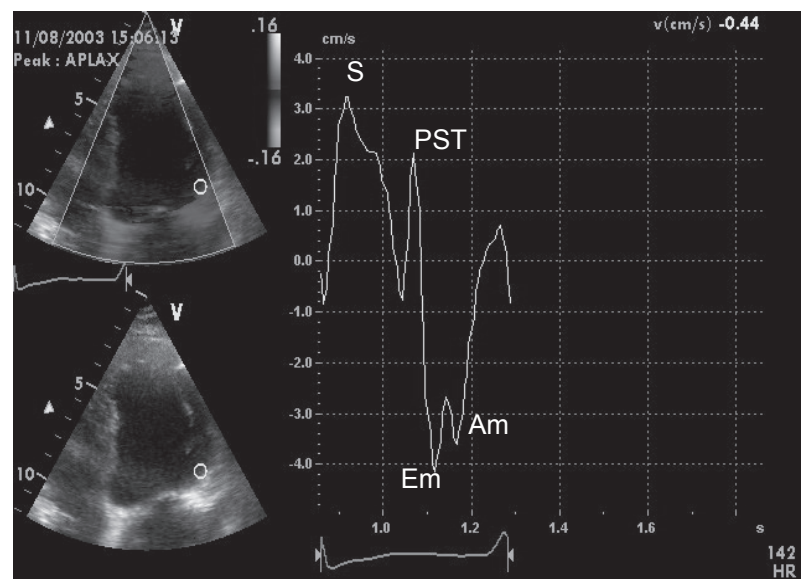

Ischemia

Figure 3 Typical regional velocity response to ischemia seen with tissue doppler imaging. In this example the basal lateral wall velocities are analyzed. At peak stress the systolic(s) and diastolic (Em and $\mathrm{Am})$ are reduced. There is also a delayed contraction seen after aortic valve closure (PST). 
CAD so a universal screening programme for this group cannot be justified, particularly as revascularization is associated with higher risk than in nondiabetics. ${ }^{39,40}$ However, certain patients may benefit such as those needing renal transplantation ${ }^{20}$ or major noncardiac surgery. ${ }^{19}$ In these groups coronary revascularization may reduce the procedural risk. ${ }^{41}$ However, diabetics with cardiac symptoms, even if atypical, and those with known or suspected CAD warrant screening and prognostic evaluation. The presence of ischemia during stress echo, especially in those with LV dysfunction at rest, permits the identification of a high risk subgroup who should be further investigated and treated accordingly. The combination of stress echocardiography and a disease specific diabetic cardiac risk score may optimize the detection of CAD and cardiac events in asymptomatic patients. ${ }^{42}$

\section{Key messages}

- Cardiovascular disease is the leading cause of death in patients with diabetes.

- DSE is a safe and reproducible technique for CAD diagnosis and risk stratification.

- Diabetic patients with a positive stress echocardiogram, especially if baseline LV systolic function is impaired, are at high cardiovascular risk.

- High risk diabetics should be referred for further cardiac investigations and potential revascularization.

\section{Disclosure}

The authors report no conflicts of interest in this work.

\section{References}

1. Kannel WB, McGee DL. Diabetes and cardiovascular risk factors: the Framingham study. Circulation. 1979;59:8-13.

2. Stamler J, Vaccaro O, Neaton JD, et al. Diabetes, other risk factors, and 12-yr cardiovascular mortality for men screened in the Multiple Risk Factor Intervention Trial. Diabetes Care. 1993;16:434-44.

3. Fuller JH, Shipley MJ, Rose G, et al. Mortality from coronary heart disease and stroke in relation to degree of glycaemia: the Whitehall study. Br Med J (Clin Res Ed). 1983;287:867-70.

4. Heyndrickx C, Baic H, Nelkins P. Depression of regional blood flow and wall thickening after brief coronary occlusion. Am J Physiol. 1978;234: H653-60.

5. Picano E, Mathias W Jr, Pingitore A, et al. Safety and tolerability of dobutamine-atropine stress echocardiography: a prospective, multicentre study. Echo Dobutamine International Cooperative Study Group. Lancet. 1994;344:1190-2.

6. Cerqueira MD, Weissman NJ, Dilsizian V, et al. American Heart Association Writing Group on Myocardial Segmentation and Registration for Cardiac Imaging. Standardized myocardial segmentation and nomenclature for tomographic imaging of the heart. A statement for healthcare professionals from the Cardiac Imaging Committee of the Council on Clinical Cardiology of the American Heart Association. Int J Cardiovasc Imaging. 2002;18:539-42.
7. Armstrong WF, Pellikka PA, Ryan T, et al. Stress echocardiography: recommendations for performance and interpretation of stress echocardiography. Stress Echocardiography Task Force of the Nomenclature and Standards Committee of the American Society of Echocardiography. J Am Soc Echocardiogr. 1998;11:97-104.

8. Segar DS, Brown SE, Sawada SG, et al. Dobutamine stress echocardiography: correlation with coronary lesion severity as determined by quantitative angiography. J Am Coll Cardiol. 1992;19:1197-202.

9. Gunalp B, Dokumaci B, Uyan C, et al. Value of dobutamine technetium-99m-sestamibi SPECT and echocardiography in the detection of coronary artery disease compared with coronary angiography. J Nucl Med. 1993;34:889-94.

10. Picano E, Lattanzi F, Distante A, et al. Role of myocardial oxygen consumption in dipyridamole-induced ischemia. Am Heart J. 1989;118:314-9.

11. Picano E, Lattanzi F, Orlandini A, et al. Stress echocardiography and the human factor: the importance of being expert. J Am Coll Cardiol. 1991;17:666-9.

12. Hoffmann R, Lethen H, Marwick T, et al. Analysis of interinstitutional observer agreement in interpretation of dobutamine stress echocardiograms. J Am Coll Cardiol. 1996;27:330-6.

13. Picano E, Bedetti G, Varga A, et al. The comparable diagnostic accuracies of dobutamine-stress and dipyridamole-stress echocardiographies: a meta-analysis. Coron Artery Dis. 2000;11:151-9.

14. Sawada SG, Segar DS, Ryan T, et al. Echocardiographic detection of coronary artery disease during dobutamine infusion. Circulation. 1991;83:1605-14.

15. Pellerin D, Brecker S. A step further in inter - institutional agreement in interpretation of dobutamine stress echocardiograms. Eur Heart J. 2002;23:768-71.

16. McCully RB, Roger VL, Mahoney DW, et al. Outcome after normal exercise echocardiography and predictors of subsequent cardiac events: follow-up of 1,325 patients. J Am Coll Cardiol. 1998;31:144-9.

17. Chuah SC, Pellikka PA, Roger VL, et al. Role of dobutamine stress echocardiography in predicting outcome in 860 patients with known or suspected coronary artery disease. Circulation. 1998;97:1474-80.

18. Pingitore A, Picano E, Varga A, et al. Prognostic value of pharmacological stress echocardiography in patients with known or suspected coronary artery disease: a prospective, large-scale, multicenter, head-to-head comparison between dipyridamole and dobutamine test. Echo-Persantine International Cooperative (EPIC) and Echo-Dobutamine International Cooperative (EDIC) Study Groups. J Am Coll Cardiol. 1999;34:1769-77.

19. Poldermans D, Arnese M, Fioretti PM, et al. Improved cardiac risk stratification in major vascular surgery with dobutamine-atropine stress echocardiography. J Am Coll Cardiol. 1995;26:648-53.

20. Sharma R, Pellerin D, Gaze DC, et al. Dobutamine stress echocardiography and the resting but not exercise electrocardiograph predict severe coronary artery disease in renal transplant candidates. Nephrol Dial Transplant. 2005;20:2207-14.

21. Al-Mansour HA, Mulvagh SL, Pumper GM, et al. Usefulness of harmonic imaging for left ventricular opacification and endocardial border delineation by Optison. Am J Cardiol. 2000;85:795-9, A10.

22. Dolan MS, Riad K, El-Shafei A, et al. Effect of intravenous contrast for left ventricular opacification and border definition on sensitivity and specificity of dobutamine stress echocardiography compared with coronary angiography in technically difficult patients. Am Heart J. 2001;142:908-15.

23. Spencer KT, Grayburn PA, Mor-Avi V, et al. Myocardial contrast echocardiography with power Doppler imaging. Am J Cardiol. 2000;86:479-81.

24. Cortigiani L, Rigo F, Gherardi S, et al. Additional prognostic value of coronary flow reserve in diabetic and non diabetic patients with negative dipyridamole stress echocardiography by wall motion criteria. $\mathrm{J} \mathrm{Am}$ Coll Cardiol. 2007;50:1354-61.

25. Cain P, Baglin T, Case C, et al. Application of tissue Doppler to interpretation of dobutamine echocardiography and comparison with quantitative coronary angiography. Am J Cardiol. 2001;87:525-31. 
26. Jamal F, Kukulski T, Strotmann J, et al. Quantification of the spectrum of changes in regional myocardial function during acute ischemia in closed chest pigs: an ultrasonic strain rate and strain study. J Am Soc Echocardiogr. 2001;14:874-84.

27. Ford ES, DeStefano F. Risk factors for mortality from all causes and from coronary heart disease among persons with diabetes. Findings from the National Health and Nutrition Examination Survey I Epidemiologic Follow-up Study. Am J Epidemiol. 1991;133:1220-30.

28. Haffner SM, Lehto S, Rönnemaa T, et al. Mortality from coronary heart disease in subjects with type 2 diabetes and in nondiabetic subjects with and without prior myocardial infarction. $N$ Engl J Med. 1998;339:229-34.

29. Pan WH, Cedres LB, Liu K, et al. Relationship of clinical diabetes and asymptomatic hyperglycemia to risk of coronary heart disease mortality in men and women. Am J Epidemiol. 1986;123:504-16.

30. Naka M, Hiramatsu K, Aizawa T, et al. Silent myocardial ischemia in patients with non-insulin-dependent diabetes mellitus as judged by treadmill exercise testing and coronary angiography. Am Heart J. 1992;123:46-53.

31. Bates JR, Sawada SG, Segar DS, et al. Evaluation using dobutamine stress echocardiography in patients with insulin-dependent diabetes mellitus before kidney and/or pancreas transplantation. Am J Cardiol. 1996;77:175-9.

32. D'Andrea A, Severino S, Caso P, et al. Prognostic value of pharmacological stress echocardiography in diabetic patients. Eur $J$ Echocardiogr. 2003;4:202-8.

33. Elhendy A, Arruda AM, Mahoney DW, et al. Prognostic stratification of diabetic patients by exercise echocardiography. J Am Coll Cardiol. 2001;37:1551-7.

34. Marwick TH, Case C, Sawada S, et al. Use of stress echocardiography to predict mortality in patients with diabetes and known or suspected coronary artery disease. Diabetes Care. 2002;25:1042-8.
35. Sharma R, Pellerin D, Gaze DC, et al. Dobutamine stress echocardiography and the resting but not exercise electrocardiograph predict severe coronary artery disease in renal transplant candidates. Nephrol Dial Transplant. 2005;20:2207-14.

36. Feringa HH, Karagiannis SE, Vidakovic R, et al. The prevalence and prognosis of unrecognised myocardial infarction and silent myocardial ischaemia in patients undergoing major vascular surgery. Coron Artery Dis. 2007;18:571-6.

37. Anand DV, Lim E, Hopkins D, et al. Risk stratification in uncomplicated type 2 diabetes: prospective evaluation of the combined use of coronary artery calcium imaging and selective myocardial perfusion scintigraphy. Eur Heart J. 2006;27:713-21.

38. De Lorenzo A, Lima RS, Siqueira-Filho AG, et al. Prevalence and prognostic value of perfusion defects detected by stress technetium-99m sestamibi myocardial perfusion single-photon emission computed tomography in asymptomatic patients with diabetes mellitus and no known coronary artery disease. Am J Cardiol. 2002;90:827-32.

39. Mick MJ, Piedmonte MR, Arnold AM, et al. Risk stratification for long-term outcome after elective coronary angioplasty: a multivariate analysis of 5,000 patients. J Am Coll Cardiol. 1994;24:74-80.

40. Barzilay JI, KronmaL RA, Bittner V, et al. Coronary artery disease and coronary artery bypass grafting in diabetic patients aged $\geq 65$ years (report from the Coronary Artery Surgery Study [CASS] Registry) Am J Cardiol. 1994;74:334-9.

41. Manske CL, Wang Y, Rector T, et al. Coronary revascularisation in insulin-dependent diabetic patients with chronic renal failure. Lancet 1992;340:998-1002.

42. Rakhit DJ, Downey M, Jeffries L, et al. Screening for coronary artery disease in patients with diabetes: a Bayesian strategy of clinical risk evaluation and exercise echocardiography. Am Heart J. 2005;150:1074-80. 
